\title{
Germanica
}

45 | 2009

La référence à l'antique dans la pensée, la littérature et les arts dans les pays germanophones au $\mathrm{XX}^{\mathrm{e}}$ siècle

\section{„Prometheus verläßt das Theater“ Zur Geschichte eines Mythos in der DDR-Kultur}

«Prometheus leaves the theater». A cultural history of a myth in Eastern

Germany

"Prométhée quitte le théâtre ». Histoire d'un mythe dans la culture de la RDA

Hans Joachim Kertscher

\section{CpenEdition}

Journals

Édition électronique

URL : http://journals.openedition.org/germanica/816

DOI : 10.4000/germanica.816

ISSN : 2107-0784

Éditeur

Université de Lille

Édition imprimée

Date de publication : 30 décembre 2009

Pagination : 59-72

ISBN : 978-2-913857-24-7

ISSN : 0984-2632

Référence électronique

Hans Joachim Kertscher, " „Prometheus verläßt das Theater“ Zur Geschichte eines Mythos in der DDR-Kultur », Germanica [En ligne], 45 | 2009, mis en ligne le 01 décembre 2011, consulté le 06 octobre 2020. URL : http://journals.openedition.org/germanica/816 ; DOI : https://doi.org/10.4000/germanica. 816

Ce document a été généré automatiquement le 6 octobre 2020.

(c) Tous droits réservés 


\title{
„Prometheus verläßt das Theater“ Zur Geschichte eines Mythos in der DDR-Kultur
}

\author{
«Prometheus leaves the theater». A cultural history of a myth in Eastern \\ Germany \\ "Prométhée quitte le théâtre ». Histoire d'un mythe dans la culture de la RDA
}

Hans Joachim Kertscher

1 Zunächst einige Vorbemerkungen: Aus dem Reservoir antiker Mythen, die bis in die Gegenwart hinein wirksam geblieben sind, weist nachgerade der Prometheus-Mythos ${ }^{1}$ eine außerordentliche Wirkmächtigkeit auf. Er fand, vor allem nachhaltig vermittelt durch Goethes Ode Prometheus von 1774, eine singuläre Wirkung in der Kulturgeschichte. Das hängt u.a. damit zusammen, dass diese Figur durch eine Reihe von Attributen gekennzeichnet ist, die insgesamt deren Ambivalenz ausmachen. Prometheus galt und gilt vielen als das Sinnbild für Fortschritt, für Risikobereitschaft, für Schöpfertum etc. Anderen hingegen ist er suspekt und bedrohlich, weil seine Taten mit einem Fluch beladen sind. Der Grund für diese heterogene Sicht auf den Mythos ist darin zu suchen, dass sich die Rezeption dieses Mythos in der Kulturgeschichte nicht einsträngig vollzog, sondern im Wesentlichen in drei Traditionslinien, deren Ursprünge die Namen Hesiod, Aischylos und Platon markieren. Hinzu kommt noch eine mündlich tradierte und schließlich von Ovid in den Metamorphosen (entstanden in den Jahren $1 \mathrm{v}$. Chr. bis etwa $10 \mathrm{n}$. Chr.) schriftlich fixierte Variation des Mythos, in der der Titanensohn als Menschenbildner figuriert.

Die Traditionslinien weisen signifikant unterschiedene Sichtweisen auf das mythische Geschehen auf. Die älteste überlieferte Variante des Prometheus-Mythos ist in Hesiods um 700 v. Chr. entstandener Theogonie bzw. in dessen Werken und Tagen zu finden. Seine Quellen sind nicht bekannt, es ist wahrscheinlich, dass hier mündlich tradierte Geschichten zusammengefasst sind, die, mit einigen Ergänzungen, Wertungen, auch Aktualisierungen versehen, zu vollständigen Texten zusammengefasst wurden. Hesiod 
versucht hier, die Entstehung der Welt, des Götter- und des Menschengeschlechts in einen großen Sinnzusammenhang $\mathrm{zu}$ bringen. Er geht von der Vorstellung eines Goldenen Zeitalters aus, in dem sich alles in einem harmonischen Zustand befand. Dieses sei von einem Eisernen Zeitalter abgelöst worden, in dem Drangsal, Elend und Krankheiten auf der Tagesordnung stehen. Als Ursache für diesen unsäglichen Zustand sieht Hesiod den Einfluss des Titanensohns Prometheus. Der habe den Göttern das Feuer gestohlen und dieses den Menschen überantwortet. Erzürnt über diese Tat rächten sich die Olympier, indem sie mit der Frau Pandora den Menschen jene Übel brachten. Prometheus hingegen wurde mit der Fesselung an den kaukasischen Felsen bestraft - und zwar, so Hesiod, völlig zu Recht. Was hier mitgeteilt wird, ist die Geschichte eines Dichters, der seine Landsleute, die böotischen Bauern, durch eine kleine Schicht Adliger hemmungslos ausgebeutet sieht. Ein Ende dieser Situation scheint nicht in Sicht zu sein.

3 Fasste Hesiod den Mythos noch in seiner religiösen Determination, die ein theoretisches Instrumentarium zur Daseinsbewältigung zu bieten schien, auf, erfährt dieser etwa 200 Jahre später eine Modifikation dahingehend, dass, bedingt durch Kolonisierungstätigkeiten im Mittelmeerraum, durch Schifffahrt und Handel, durch das Aufkommen des Geldes etc. kleinbürgerliche Schichten immer stärker an wirtschaftlicher und damit auch politischer Einflussnahme in den griechischen Poleis gewinnen. Ein Ausdruck dafür sind die in Athen veranstalteten Prometheia, die Fackelläufe der Handwerker zu Ehren des Titanensohns. Philosophie, Kunst und Wissenschaft reflektieren diesen Prozess auf die ihnen adäquate Weise. Eine dem Hesiodschen Typus diametral entgegengesetzte Gestaltung des Prometheus ist in Aischylos' Tragödientrilogie Prometheus zu finden. ${ }^{2}$ Obwohl davon nur Der gefesselte Prometheus auf uns gekommen ist, kann durchaus festgestellt werden, dass hier ein völlig neues Bild des Titanen entwickelt wird. Er ist nicht mehr der zu Recht Bestrafte, sondern derjenige, der gegen jegliche Autorität rebelliert, der mutig zu seinen Taten steht und diese gegenüber den Göttern preist. Damit wird auch den Menschen ein weitaus größerer Radius sozialer Aktivitäten zugebilligt. Das ihnen überlassene Feuer ermöglicht kulturelle Fortschritte in ungeahnter Weise. Prometheus wird zur Symbolfigur des menschlichen Strebens nach Freiheit und Unabhängigkeit.

Etwa 100 Jahre später relativiert Platon die Erwartungshaltungen, die sich an den prometheischen Taten ausgerichtet hatten. Platon vermittelt einen Dialog zwischen den Philosophen Protagoras und Sokrates (um 388 v. Chr.), in dem es um die Lehrbarkeit der Staatskunst, der politike techne geht. Protagoras erzählt hier die Geschichte des Prometheus-Bruders Epimetheus, der bei der Ausstattung der Lebewesen die Menschen vergaß. Prometheus hilft aus, stiehlt das Feuer, die Kunstfertigkeit des Hephaistos und die Weisheit der Athene (insgesamt: die techne) und schenkt diese den Menschen, die sich nun auf der Welt einrichten können. „Auf diese Art bekam der Mensch die Weisheit, welche er zur Erhaltung seines Lebens nötig hatte; aber die Weisheit, welche zum bürgerlichen Leben nötig ist, oder die Politik, hatte er noch nicht“. Der Zugang zur Staatskunst blieb Prometheus versperrt. „Schamhaftigkeit und Gerechtigkeit ${ }^{{ }_{3}}$ ließ Zeus den Menschen durch Hermes zukommen. Indem Protagoras dem Prometheus lediglich den Zugriff auf die techne, nicht aber auf die politike techne lässt, verweist er auf die Unzulänglichkeit des titanischen Handelns und wertet gleichzeitig die Möglichkeiten des göttlichen Eingreifens auf. Protagoras argumentiert so angesichts eines zunehmenden Niedergangs der griechischen Polisdemokratie, deren Grundlage, die Sklavenhalterei, sich immer stärker als ein 
Hemmnis gesellschaftlichen Fortschritts erwiesen hatte. Dies wird von dem Philosophen mit wachem Blick registriert und das einstige Symbol partiell kritisiert.

Vor allem die beiden zuletzt genannten Traditionen spielen in der Rezeption des Prometheus-Mythos in Kunst und Wissenschaft eine nicht gering zu schätzende Rolle. Auf den aischyleischen Prometheus berief sich, wer sich von jeglicher Vorherrschaft befreien wollte. Er galt jenen gesellschaftlichen Klassen, die sich im Emanzipationskampf befanden, als kühner Rebell, als Freund der Unterdrückten, als der Produktive, schöpferisch Tätige und wurde somit zu deren Symbol- bzw. Identifikationsfigur. Nach der Konsolidierung der Macht, aber vor allem angesichts signifikanter Verfallserscheinungen derselben, wird die Sicht auf die einstige Vorbildfigur zunehmend kritischer. Hervorgehoben wird nunmehr die Ambivalenz des Prometheischen. Schöpfertum erscheint als fragwürdig, technischer Fortschritt als dem Menschen feindlich. Schließlich werden die Mächtigen und deren Institutionen selbst in Frage gestellt.

Das lässt sich deutlich anhand der Geschichte des, wie er sich selbst apostrophierte, ersten deutschen Arbeiter- und Bauernstaates', der Deutschen Demokratischen Republik, die von 1949 bis 1990 existierte, nachweisen. Gestützt auf Karl Marx’ Dissertation von 1841, in der er das „Bekenntnis“ des aischyleischen Prometheus „gegen alle himmlischen und irdischen Götter, die das menschliche Selbstbewußtsein nicht als die oberste Gottheit anerkennen“ wollen, zum Prüfstein jeglicher Philosophie erhebt („Prometheus ist der vornehmste Heilige und Märtyrer im philosophischen Kalender “4), wird der Mythos vornehmlich als Symbol der Selbstbefreiung des Proletariats, als Leitbild für eine neue Gesellschaft genutzt. Bereits 1940, in der Zeit seines Exils in der Sowjetunion, entwirft Johannes R. Becher, der spätere Kulturminister der DDR, in seinem Gedicht Prometheus die Vision einer glücklichen Zukunft, in der die Menschen sich befreien werden:

Es reichten ihre Arme schon so weit,

Und des Titanen Stimme ließ sie ahnen:

Die Götterfeste stürzt. Es naht die Zeit

Der Menschenmacht! Die Herrschaft der Titanen [...]. ${ }^{5}$

7 Damit war eine Grundlage für jene Prometheus-Aneignung gegeben, die für lange Zeit zum Paradigma in Kunst und Literatur der DDR, wiewohl in verschiedenerlei Modifikation, werden sollte. Ständig präsent war dabei, wie schon bei Becher, der Prometheus der gleichnamigen Ode Goethes von 1773, die mit folgenden Versen endet:

Hier sitz ich, forme Menschen

Nach meinem Bilde

Ein Geschlecht das mir gleich sei

$\mathrm{Zu}$ leiden, weinen

Genießen und zu freuen sich

Und dein nicht zu achten

Wie ich! ${ }^{6}$

Menschenformung und Negation des Alten standen auch im Vordergrund einer Rede, die Wilhelm Girnus, der Staatssekretär für das Hochschulwesen der DDR, 1957 zum 40. Jahrestag der Russischen Oktoberrevolution hielt: „Wir vermögen heute in dieser ergreifenden archaischen Titanengestalt das mythisch verhüllte Selbstbildnis der Menschheit und ihr jugendlich aufbrechendes, sich an sich selbst berauschendes Schöpfungsbewußtsein zu entziffern, den Glauben an ihre Zukunft, ihr erfinderisches Genie, ihre alles durchdringende Kraft der Erkenntnis. Es ist das Bild, das sich die 
Menschheit in ihrer historischen Morgendämmerung davon macht". Prometheisches verkörpere "die titanische Stimme menschlicher Macht, die vor nichts kapituliert und in sich die Kraft fühlt, die Welt aus den Angeln zu heben und sie nach ihrem Bilde zu formen".?

Vom August 1969 bis zum Januar 1970 initiierte das Zentralorgan der SED, die Zeitung Neues Deutschland, eine Leserdiskussion zum Thema „Was ist der Mensch? Kain oder Prometheus?", zu der Harald Wessel, der Leiter der Abteilung Wissenschaft dieses Blattes, die Zielrichtung absteckte, indem er die aischyleische Tradition des Prometheus-Mythos referiert und dann betont: „Dieses humanistische Bild ist heute keineswegs bloße Legende mehr. Die Kraft der sozialistischen Welt gibt dem prometheischen Drang der Menschheit den nötigen Rückhalt “. ${ }^{8}$ Entlang dieser Vorgabe verlief denn auch die ,Diskussion', die ihren Tiefpunkt in einem Beitrag von B. Böckmann erreichte: „Erziehen müssen wir den Prometheus natürlich so, daß er lieben und hassen kann. Er muß es, weil er nötigenfalls mit dem Gewehr in der Hand die von ihm geliebte sozialistische Heimat verteidigen muß. Auch ein Prometheus muß töten, wenn er dazu vom Klassenfeind gezwungen wird“. 9

Wenige Monate zuvor hatte der Berliner Ästhetiker Wolfgang Heise derlei undifferenziert vorgetragenen Äußerungen gegenüber sein Missfallen ausgedrückt: „Gewiß, das Proletariat bedarf nicht heroischer Illusionen, um sich über den Inhalt seiner Revolution hinwegzutäuschen. Es bedarf vielmehr des Abbaus aller Illusion, der klaren Bewußtheit seines eigenen Inhalts, um historischer Akteur zu sein. Es muß sich nicht selbst in Gestalt des Prometheus oder Herakles monumentalisieren, gerade weil es deren perspektivische geschichtliche Gehalte realisiert ${ }^{\text {" }}{ }^{10} \mathrm{Er}$ hätte sich in diesem Zusammenhang auch auf den deutschen Aufklärer Christoph Martin Wieland berufen können, der sich von Jugend an mit dem Prometheus-Stoff beschäftigt hatte und in einem fiktiven Gespräch mit einem Pfarrer vom Schriftsteller forderte, seinen Lesern anstelle monumentaler Charaktere reale Menschen zu zeigen und vorzuführen: wie sie „unter dem Drucken, Stoßen und Anziehen so unzählig vieler auf sie wirkender mechanischer, lebendiger und geistiger Kräfte, und mitten unter so vielen Schwierigkeiten, Hindernissen und Kollisionen, Abwegen und Fährlichkeiten, wovon sie umgeben sind - es anzufangen haben, um so weise, fromm, gerecht und gut zu seyn, als es unter besagten Umständen möglich ist." ${ }^{11}$ Es sei durchaus vorstellbar, „daß die Geschichtschreiber der Menschheit nützlicher sind als die Prometheen, die uns neue Menschen nach ihrem eigenen Bilde schnitzeln ". ${ }^{12}$

11 Heises Äußerungen signalisierten einen Wandel im Umgang mit dem PrometheusThema, der nicht zuletzt auf zunehmende Widersprüche innerhalb der gesellschaftlichen Situation in der DDR zurückzuführen war. Der Bau der Mauer von 1961, euphemistisch mit ,Antifaschistischer Schutzwall' umschrieben, und die damit einhergehende Abschottung der DDR-Bevölkerung hatte nicht, wie vielleicht von den Parteioberen erhofft, eine souveräne und stetige Wirtschaftsentwicklung zur Folge, sondern es dominierten weiterhin Misswirtschaft und ein desaströser Umgang mit Ressourcen und Umwelt. Rainer Kirsch schrieb dazu später ein sarkastisches Distichon:

Groß in Gesängen rühmten die Alten den Schaffer Prometheus

Weil er das Feuer uns gab; wir heute schlucken den Rauch. ${ }^{13}$

Vor allem aber machten sich in der Kulturpolitik Anzeichen restriktiver Maßnahmen bemerkbar. Waren unmittelbar nach 1961 noch literarische Produktionen wie Christa Wolfs Geteilter Himmel oder Brigitte Reimanns Ankunft im Alltag möglich gewesen, die 
Probleme des DDR-Alltags realistisch beschrieben hatten, zog Erich Honecker, der später Vorsitzender des Staatsrates der DDR werden sollte, in seiner Rede auf dem 11. Plenum der SED vom Dezember 1965 einen Schlussstrich unter derlei Aktivitäten. Verbote von Filmen, musikalischen Werken, literarischen Texten, Ausschlüsse unliebsamer Künstler aus deren Berufsverbänden bzw. aus der SED folgten und lähmten so das gesamte geistige und kulturelle Leben in der DDR. Die 1976 erfolgte Ausbürgerung des Liedermachers Wolf Biermann aus der DDR war der negative Höhepunkt einer verfehlten Kulturpolitik. Unter derlei Vorzeichen konnten nur noch verblendete Künstler oder Opportunisten den sozialistischen Menschen als den neuen Prometheus feiern. Sowohl in der Literatur als auch in der bildenden Kunst und der Musik wurden nunmehr Stimmen laut, die hinsichtlich der Prometheus-Aneignung nicht mehr prononciert auf die aischyleische Tradition setzten. Noch mit Bezug auf Aischylos, aber in deutlicher Distanz zu ihm, lässt Volker Braun sein PrometheusGedicht von 1967 enden:

Bedroht, aber nicht gedrillt

Sieht mich der Tag

Der widerstrahlt

Wenn wir unser Feuer tragen

In den Himmel..$^{14}$

Offensichtlich war es die gründliche Beschäftigung mit dem Werk Bertolt Brechts, die bei einigen Schriftstellern neue Denk- und Gestaltungsstrukturen evozierte. Der hatte nämlich, zeitlich etwa parallel zu dem Antipoden Becher, d.h. zwischen der Arbeit am Leben des Galilei und dem Fragment gebliebenen Leben des Einstein, Studien zu einem Drama Die Verurteilung des Prometheus betrieben. Im Arbeitsjournal findet sich dazu unter dem 2. Oktober 1945 folgende Notiz: „Erwäge einen Prometheus. Die Götter sind unwissend und bösartig, schlau im Erpressen von Opfern, lebend von den Fetten des Lands. Prometheus erfindet das Feuer und übergibt es verbrecherischerweise den Göttern. Sie fangen und fesseln ihn, damit er nicht den Menschen sein Feuer ausliefern kann. Von diesem Feuer erfährt er lange nichts, dann sieht er rote Feuersbrünste am Horizont: die Götter haben es benutzt, Menschen zu brandschatzen. Die Götter nur als Chor auftretend. " ${ }^{15}$ Diese kurzen Notizen verdeutlichen, dass Brecht, konfrontiert mit der bedenkenlosen Forcierung der Herstellung einer Atombombe durch amerikanische Wissenschaftler, einen in der platonischen Tradition stehenden Prometheus im Auge hatte. Ernst Schumacher beschreibt diesen „als Helfer der Menschen und Kollaborateur mit den Oberen ". ${ }^{16}$ In Brechts Nachlass befinden sich Variationen des Vorhabens: So warnen in der einen die Götter den Protagonisten, den Menschen das Feuer zu überantworten, da sie nicht reif dafür seien. Dessen Knecht jedoch erfindet, aus Furcht vor den Schrecknissen der Natur, das Feuer selbst mit Hilfe von Reibhölzern. In einer anderen sind es die Menschen, die den Titanen fesseln und in einer weiteren ist von dessen Vertrauen, dass die Menschen das Feuer in produktiver Weise verwenden werden, die Rede. Brechts Haltung zu diesem Stoff war offenbar so ambivalent wie der Stoff selbst - und damit hinsichtlich einer weiteren Gestaltung für ihn uninteressant geworden.

14 Nicht so für Heiner Müller, der Brechts Hinweis auf die Ambivalenz der platonischen Prometheus-Gestalt ernst nahm. Auf diese hatte bereits Goethe, angesichts beunruhigender Wendungen im postrevolutionären Frankreich und bedenklicher Erfahrungen im Zusammenhang mit depravierenden Folgeerscheinungen der 
Industriellen Revolution in einigen europäischen Ländern, in seinem Festspiel Pandora von 1808 verwiesen, das er mit folgenden Versen enden lässt:

Groß beginnet ihr Titanen; aber leiten

$\mathrm{Zu}$ dem ewig Guten, ewig Schönen,

Ist der Götter Werk; die laßt gewähren! ${ }^{17}$

Zuvor (1783) hatte er schon in dem Geburtstags-Gedicht Ilmenau, das seinem Schützling Karl August galt, mit Versen, die im Zentrum des Textes situiert sind, die Folgen prometheischen Tuns kritisch reflektiert:

Ließ nicht Prometheus selbst die reine Himmels Glut

Auf frischen Ton vergötternd niederfließen

Und konnt er mehr als irdisch Blut

durch die belebten Adern gießen?

Ich brachte Feuer vom Altar

Was ich entzündet ist nicht reine Flamme [... ${ }^{18}$

16 Auch Georg Friedrich Wilhelm Hegel bemühte in seinen Vorlesungen über die Ästhetik, die er zwischen 1817 und 1829 hielt, den Protagoras-Dialog und meinte: „Das Sittliche, Rechtliche hat Prometheus den Menschen nicht gegeben, sondern nur die List gelehrt, die Naturdinge zu besiegen und zum Mittel menschlicher Befriedigung zu gebrauchen. Das Feuer und die Geschicklichkeiten, die sich des Feuers bedienen, sind nichts Sittliches in sich selbst, ebenso wenig die Webekunst, sondern treten zunächst nur in den Dienst der Selbstsucht und des Privatnutzens, ohne auf das Gemeinsame des menschlichen Daseins und das Öffentliche des Lebens Bezug zu haben. Weil Prometheus nichts Geistigeres und Sittlicheres dem Menschen zuzuteilen im Falle war, gehört er auch nicht dem Geschlecht der neuen Götter an, sondern der Titanen “. ${ }^{19}$ Ersteren hingegen sei Prometheus' Befreier Herakles zuzurechnen: „Herakles wird zur bevorzugten Gestalt, weil sein Handeln als Modell dienlich sein kann für die schnelle Verwirklichung gestellter Arbeiten". ${ }^{20}$

In Heiner Müllers Intermedium Befreiung des Prometheus, das in die 1972 fertiggestellte Dramatisierung von Fjodor Gladkows Roman Zement (1925) eingebaut ist, wird der Titanensohn als ein Opportunist vorgestellt, „der den Menschen den Blitz ausgeliefert, aber sie nicht gelehrt hatte, ihn gegen die Götter zu gebrauchen, weil er an den Mahlzeiten der Götter teilnahm, die mit den Menschen geteilt weniger reichlich ausgefallen wären ". ${ }^{21}$ Prometheus, der seine Befreiung durch Herakles halbherzig über sich ergehen lässt und gegenüber dem Olymp greinend seine Unschuld daran betont, nimmt erst nach dem Tod der Götter „auf der Schulter seines Befreiers [...] die Haltung des Siegers ein“. ${ }^{22}$ Die Anspielung auf intellektuelles Gebaren unter problematischen, dann aber wieder günstig scheinenden Umständen, ist unübersehbar. Der Text des Intermediums wird von dem bürgerlichen Ingenieur Kleist, den es für den sozialistischen Aufbau zu gewinnen gilt, gesprochen. Die Verbindung PrometheusKleist und deren dramatische Realisierung verweisen auf die signifikanten Widersprüche, unter denen sich dieser Aufbau vollzieht. Der Befreier Herakles steht für die revolutionäre Arbeiterklasse, die zur Durchsetzung ihrer Interessen auf die Fähigkeiten des Ingenieurs angewiesen ist. Sie muss ihn aus seinen bürgerlichen Bindungen befreien, um ihn benutzen zu können. Darüber hinaus - und hier gewinnt der Vorgang seine bittere Ironie - ist sie gezwungen, ihm Privilegien, u.a. die Rolle des Siegers zu spielen, zukommen zu lassen. Es ist Wolfgang Heise zuzustimmen, wenn er konstatiert: „Das ist weder vorgängiges noch nachträgliches Mythologisieren [...], sondern die eigene Epoche wird gerade durch das Einbeziehen der historisch- 
mythischen Gestalten in ihrer Geschichtlichkeit erfaßt und formulierbar. Die Gegenwartsgestalt gewinnt geschichtliche Tiefendimension" “. ${ }^{23}$

Ebenfalls stärker die historischen Dimensionen des Mythos auslotend zeigt Franz Fühmann einen archaischen Prometheus. Im Zusammenhang mit Nacherzählungen mythologischer Stoffe für Kinder hatte sich der Dichter Anfang der siebziger Jahre des 20. Jahrhunderts mit mythologischen Fragen eingehend beschäftigt und die Ergebnisse am 28. Februar 1974 vor Studenten der Berliner Humboldt-Universität referiert. Ein Jahr später erschienen sie unter dem Titel Das mythische Element in der Literatur. Er berichtet da u.a. von seiner "vergeblichen Suche nach einem Ur-Prometheus“, was einer „Höllenfahrt des Gewissens“ gleichkam und in der Erkenntnis endete: „Ein Mythos, das ist der Keim und all seine Entfaltungen; gerade das Werden in stets neuer Gestaltung ist sein Leben; das Erstarren aber zu einem von nun ab als einzig gültig Bestimmten wäre sein Tod. [...] Die Treue zum Mythos erfordert Untreue gegenüber allen seinen vorhandenen Fassungen ". ${ }^{24}$ So erweist sich sein für Kinder geschriebener Prometheus als eine wohl stärker der platonischen Tradition verpflichtete mythologische Gestalt, die eher Hilflosigkeit denn Kraft und Kühnheit ausstrahlt.. Dieses Vorhaben Fühmanns blieb unvollendet. Erst 1996 hat Sigurd Schmidt den um 1973 geschriebenen zweiten Teil (fünf Teile waren insgesamt geplant) aus dem Nachlass Fühmanns unter dem Titel Prometheus. Die Zeugung publiziert. Hier nun gewinnt das Geschehen um den Protagonisten deutliche Parallelen $\mathrm{zu}$ zeitgeschichtlichen Ereignissen. Prometheus versucht, die Insel Kreta für sein Menschenpaar bewohnbar zu machen. Aus „Sorge um ihr Wohl“" ${ }^{\text {“6 }}$ baut er um deren Unterkunft einen „Schutzwall“", ${ }^{27}$ um sie von sämtlichen Einflüssen der Außenwelt fernzuhalten. So erreicht er einerseits, dass sie in ihrer Isolation vor jeglichen Fährnissen geschützt sind, andererseits aber in ihren Entwicklungsmöglichkeiten auf ein Minimum beschränkt bleiben. Prometheus schafft sich so die Gewähr, dass sie auch ihm nicht gefährlich werden können. Gemeint ist die „Sorge um seinen Besitz“. Die Menschen sollten etwas sein , „das nur ihm gehörte und das keine Selbständigkeit besitzt ${ }^{\text {“ }}{ }^{28}$ Resignierend muß sich Prometheus eingestehen: „Ein Reich der Gerechtigkeit wollte er gründen, und er war ungerechter als Kronos und Zeus! ${ }^{{ }^{29}}$ Die ironisierenden Anspielungen auf die DDR-Wirklichkeit sind unübersehbar. Offenbar war sich Fühmann im Klaren darüber, dass dieser Text die Zensur der DDR nicht passieren würde und unterließ so auch dessen Fortsetzung.

Bereits 1965, also früher als Heiner Müller, stellte Peter Hacks eine platonisch geprägte Sicht auf den Prometheus-Mythos vor. In der Bearbeitung der Vögel von Aristophanes erscheint der Titan als intellektueller Opportunist, der es stets mit den jeweils Herrschenden hält. Seine Gabe der Voraussicht erschöpft sich lediglich darin, dass er sich erst nach dem Sieg des Stärkeren zu ihm bekennt:

Doch furchtbar werden, dies auch ist vorauszusehn,

Die Kämpfe toben. Ehe die entschieden sind,

Will ich mich offen nimmermehr entscheiden. Drum

Verhüll ich klüglich mein Gesicht mit einem Schal

Und täusche Zeus' allüberblickend Späheraug

Mit einem Regendache. [...] So zu handeln ist

Voraussichtlich [Hervorh. H.-J. K.] das beste. ${ }^{30}$

20 Von dieser Sehweise ist in der Bearbeitung des Goetheschen Pandora-Festspiels (1981), in dem der Bruderzwist Prometheus-Epimetheus eine gewichtige Rolle spielt, nichts mehr zu spüren. In einem dem Drama angefügten Essay meint Hacks, dass Goethe „dem dichtenden Titanen Epimetheus Recht und dem tätigen Titanen Prometheus Unrecht“ 
gebe. „Prometheus wird in die Segensherrschaft der Pandora nicht einbezogen.“" ${ }^{31}$ Dies ist eine Lesart, die wohl an Goethes Intentionen vorbeigeht. Dessen fragmentarisch gebliebenes Festspiel hatte eine Krisensituation signalisiert: „Pandora sowohl als die Wahlverwandtschaften drücken das schmerzliche Gefühl der Entbehrung aus“, ${ }^{32}$ notiert der Dichter in den Tag- und Jahresheften. Die beiden Brüder werden im ersten Aufzug radikal auf ihre Grundzüge reduziert. Prometheus ist der Mann der Tat, Epimetheus der der Reflexion, der Kunst. Beide erweisen sich in ihrer Einseitigkeit als lebensuntüchtig. Eine Vereinigung der beiden Grundzüge deutet Goethe in der Vermählung von Phileros und Epimeleia, der Kinder der Titanen, an. Dies schien ihm ein möglicher Ausweg aus der gesellschaftlichen Krisensituation des beginnenden 19. Jahrhunderts zu sein. Lediglich eine kurze Bemerkung in den Paralipomena, die auf die Fortsetzung des Festspiels hinweisen, lässt andeutungsweise erkennen, dass eine "Verjüngung des Epimetheus“ ins Auge gefasst sei und „Pandora mit ihm emporgehoben “ ${ }^{\text {“33 }}$ werde. Grundsätzlich ändert das freilich nichts an der Grundvoraussetzung Goethes, die einzig in der Vereinigung von Prometheischem und Epimetheischem eine mögliche gesellschaftliche Perspektive aufweist. Insofern ist es Spiegelfechterei, wenn Hacks mit seiner Dramatisierung eine Korrektur der Goetheschen Konzeption vorzunehmen vorgibt: „Vom Standpunkt des reifen Sozialismus her läßt die mögliche Einheit von Tat und Entwurf als Hoffnung sich wieder schreiben“.34 Von „Hoffnung“" ${ }^{35}$ auf eine Zukunftsbewältigung der sozialistischen Gesellschaft also sind Hacks' Bearbeitungsintentionen geprägt. Sie wird durch Pandora symbolisiert: „Hoffnung, endlich nicht mehr namenlos.“" ${ }^{36}$ Die Verbindung von Epimeleia und Phileros lässt er im Gegensatz zu Goethe scheitern. Stattdessen führt er am Ende die entzweiten Brüder zusammen - allerdings auf einem neuen Niveau: Beide erkennen, dass sie sich ändern müssen. Prometheus sieht ein, dass er sich fortan auch „Des Gedankens lastrückenden Hebels“"37 bedienen müsse und für Epimetheus gilt: „Fühle genauer, traure mehr erfindungsreich.“" ${ }^{38}$ So kann Pandora, die „Hoffnung“, zurückkehren.

Von derlei Assoziationen war allerdings in dem Prometheus-Projekt, das vom Kulturbund der DDR ein Jahr später anlässlich des 150. Todestages Goethes initiiert wurde, nicht mehr allzu viel zu spüren. Schriftsteller, Musiker und bildende Künstler waren aufgefordert worden, sich in der ihnen gemäßen Weise zu ihrer PrometheusAuffassung zu äußern. Erwartet wurde ein deutliches Bekenntnis zur aischyleischen Tradition und zur sozialistischen Gesellschaft. Was dabei herauskam, war die Anzeige nicht nur einer Krisen-, sondern einer Endzeitsituation eben dieser Gesellschaft. Aus der Fülle der Beiträge sollen hier zwei herausgegriffen werden. ${ }^{39}$ Heinz Czechowski bezog sich direkt auf Goethes Ode und persiflierte diese dahingehend, dass Prometheus die Menschen nicht nach seinem Bilde, sondern nach denen der Götter geschaffen habe:

Sie [die Menschen, H.-J. K] brannten mir dankbar

All ihre Orden auf meine Brust,

Dann riefen sie: Kreuziget ihn! //

Hier stehe ich nun,

Beladen mit ihrer Schuld, und seh,

wie sie, uneins geworden,

Gierig trinken

Aus Pandoras Büchse.//

Nicht sehend geworden, 
Spotten sie mit ihren Erfindungen

auch noch des Tods [...]. ${ }^{40}$

Vor allem die bildenden Künstler wussten hier mit einer erstaunlichen Vielfalt in der Behandlung des Themas aufzuwarten. Nach einer anfänglichen Zurückhaltung gegenüber einer Prometheus-Gestaltung, von 1945 bis 1949 widmeten sich zwei Künstler in zwei Werken dem Thema, von 1950 bis 1959 wird das Thema nicht aufgegriffen, von 1960 bis 1969 lassen sich vier Werke von vier Künstlern nachweisen, von 1970 bis 1979 entstehen sieben Werke von sechs Künstlern und allein von 1980 bis 198437 Werke von 19 Künstlern. ${ }^{41}$ Eines davon war die Graphik Prometheus verlässt das Theater, das Wolfgang Mattheuer als Beitrag zu dem Projekt beisteuerte. Sie zeigt vordergründig eine nackte männliche Gestalt, die sich mit einem großen Schritt aus einem eng begrenzten Raum, der von Flammen bedroht wird und bestückt ist mit mancherlei Requisiten (nahezu alles Zitate aus früheren Arbeiten Mattheuers) und Schattenfiguren, auf eine geöffnete Tür $\mathrm{zu}$ bewegt, welche den Blick auf einen unbegrenzten Raum freigibt. Diese Arbeit gehörte wohl zu den am meisten diskutierten Beiträgen dieses Projekts. Vorangegangen war eine Bleistiftzeichnung auf Karton vom 12. September 1981, die den Titel „Prometheus verläßt das Theater oder Das Ende der Aufklärung“ erhielt. Hier sind bereits die wesentlichen Momente des später eingereichten Holzschnitts enthalten. Mattheuers Werk macht es dem Betrachter bis heute nicht leicht, eine schlüssige Lesart für das Vorgestellte $\mathrm{zu}$ finden. Zu kurz gegriffen wäre jene, die eine Anspielung auf den Exodus vieler Künstler aus der DDR sehen will. Einig war sich die Kritik darin, ${ }^{42}$ dass hier ein Künstler sein eigenes Werk in Frage stellt, das er dem Feuer überantwortet, welches er bekanntermaßen zuvor selbst gebracht hatte. Probleme des Schöpferischen sind es wohl, die im Vordergrund der künstlerischen Gestaltung stehen. Dieses zielt immer auf Kritik am Althergebrachten, auf Negation des Geschaffenen. Und Schöpferisches ist immer auch mit Mut, mit Risikobereitschaft verbunden und verlangt, dass der Schöpferische auch Verluste in Kauf zu nehmen bereit ist. Insofern entledigt sich der zu neuen Ufern Aufbrechende jeglichen Ballastes, der ihn noch mit dem Alten verbinden könnte. Nackt tritt er dem ihm noch fremden Aufgabenbereich entgegen. Das ist keine Schutzlosigkeit, sondern vielmehr die Besinnung auf die eigene schöpferische Kraft, die willens ist, sich unvoreingenommen und von allen Äußerlichkeiten befreit der anstehenden und ungewohnten Aufgabe zu widmen. Mattheuers Intentionen könnten freilich auch in Richtung einer Gestaltung in der Theatrum-mundi-Tradition angesiedelt sein. Darauf lässt der Untertitel der Bleistiftzeichnung („Ende der Aufklärung“) schließen. Aufklärung meinte ja auch, dass sich der Mensch nicht mehr als funktionierendes Glied innerhalb einer festgefügten Ordnung begreifen solle, sondern vielmehr als Subjekt, das in der Lage ist, eigenschöpferisch an der Weltgestaltung teilzunehmen. Mattheuers Graphik verweist auf eine Endsituation, in der sich die vom Menschen missbrauchte Welt nunmehr befindet. Zum Zeitpunkt der Entstehung dieses Werkes war das Wettrüsten zwischen Ost und West auf einem Höhepunkt angelangt. Prometheus verlässt fluchtartig dieses Welttheater. Das, was er der Menschheit geben konnte, die techne, reichte nicht aus, um diese Welt harmonisch gestalten zu können. Es fehlte die politike techne, die Staatskunst. Derartige Prometheus-Vorstellungen mussten den DDROberen, die sich im sicheren Besitz der weisen Staatskunst wähnten, suspekt erscheinen, ein Verbot der Veröffentlichung des Projekts folgte auf dem Fuß. Acht Jahre später existierte die DDR nicht mehr. 


\section{NOTES}

1. Generell zur Prometheus-Problematik vgl. Kertscher, Hans-Joachim: Prometheus. Mythos und Stoff in der Kulturgeschichte. Diss. habil. [masch.]. MLU Halle 1987.

2. Allenthalben wurde und wird die Autorschaft Aischylos' am Gefesselten Prometheus bestritten, so u.a. von Mark Griffith; vgl. ders.: The Authenticity of „Prometheus Bound“. Cambridge 1977.

3. Platon: Protagoras. Frankfurt (M.)/Hamburg 1955, S. $23 f$.

4. Marx, Karl: Differenz der demokritischen und epikureischen Naturphilosophie. In: Marx, Karl/ Engels, Friedrich: Werke. Ergänzungsband. Erster Teil. Berlin 1968, S. $262 f$.

5. Becher, Johannes R.: Prometheus. In: Ders.: Gedichte. 1936-1941. Berlin/Weimar 1966, S. 612.

6. Goethe, Johann Wolfgang: Prometheus. In: Ders.: Gedichte 1756-1799. Hg. v. Karl Eibl. Frankfurt/M. 1998, S. 204.

7. Girnus, Wilhelm: Die Befreiungsstunde des prometheischen Geistes. Berlin 1957, S. $10 \mathrm{ff}$.

8. Wessel, Harald: Was ist der Mensch? Kain oder Prometheus? In: Neues Deutschland, 13.9.1969,

S. 10 .

9. Vgl. ebd., 24.1.1970, S. 10.

10. Heise, Wolfgang: Bemerkungen zur Funktion und Methode der Antikenrezeption in der klassischen deutschen Literatur. In: Wissenschaftliche Zeitschrift der Friedrich-SchillerUniversität Jena. Gesellschafts- und sprachwissenschaftliche Reihe. H. 4 (1969), S. 56.

11. Wieland, Christoph Martin: Unterredungen mit dem Pfarrer von ***. In: Wielands Gesammelte Schriften [Akademie-Ausgabe]. I. Abt., 14. Bd. Hg. v. Wilhelm Kurrelmeyer. Berlin 1928, S. 58.

12. Ebd., S. 61.

13. Kirsch, Rainer: Das Ende vom Lied. In: Prometheus 1982. Unbeliebte Kunst aus der DDR. Hg. v. Roland Rittig u. Rüdiger Ziemann. Halle/Zürich 1995, S. 19.

14. Braun, Volker: Prometheus. In: Ders.: Wir und nicht sie. Gedichte. Halle 1976, S. 64.

15. Brecht, Bertolt: Journale 2. In: Ders.: Werke. Große kommentierte Berliner und Frankfurter Ausgabe. Berlin/Weimar/Frankfurt (M.) 1995, S. 233.

16. Schumacher, Ernst: Drama und Geschichte. Bertolt Brechts „Leben des Galilei“ und andere Stücke. Berlin 1965, S. 319.

17. Goethe, Johann Wolfgang: Pandora. Ein Festspiel. In: Ders.: Berliner Ausgabe. Poetische Werke. Bd. 6. Berlin/Weimar 1980, S. 447.

18. Goethe, Johann Wolfgang: [Ilmenau]. In: Ders.: Gedichte (wie Anm. 6), S. 266.

19. Hegel, Friedrich: Ästhetik. Hg. v. Friedrich Bassenge. Bd. 1. Berlin/Weimar 1976, S. 431 f.

20. Ebd., S. 446.

21. Müller, Heiner: Zement. In: Ders.: Stücke. Berlin 1975, S. 343.

22. Ebd.

23. Heise, Wolfgang: Zum Kontext der Frage nach der Antike. In: Weimarer Beiträge. H. 1 (1985), S. $33 \mathrm{f}$.

24. Fühmann, Franz: Das mythische Element in der Literatur. In: Ders.: Erfahrungen und Widersprüche. Versuche über Literatur. Rostock 1975, S.170-174.

25. Vgl. Fühmann, Franz: Prometheus. Die Titanenschlacht. Berlin 1974.

26. Fühmann, Franz: Prometheus. Die Zeugung. Hg. v. Sigurd Schmidt. Rostock 1996, S. 24.

27. Ebd., S. 17.

28. Ebd., S. 24.

29. Ebd., S. 25.

30. Hacks, Peter: Die Vögel. In: Ders.: Stücke nach Stücken 2. Berlin/Weimar 1983, S. 38.

31. Hacks, Peter: Saure Feste. Nachwort zu „Pandora“. In: Ders.: Essais. Leipzig 1984, S. 438. 
32. Goethe, Johann Wolfgang: Tag- und Jahreshefte. In: Ders.: Sämtliche Werke. I. Abt., Bd. 17. Hg. v. Irmtraud Schmid. Frankfurt/M. 1994, S. 215.

33. Goethe, Pandora (wie Anm. 17), S. 450.

34. Hacks, Feste (wie Anm. 31), S. 438.

35. Bekanntlich war es die Hoffnung, die die Pandora Hesiods in ihrem Gefäß zurückbehielt.

36. Hacks, Peter: Pandora. In: Ders.: Stücke (wie Anm. 30), S. 233.

37. Ebd., S. 234.

38. Ebd., S. 227.

39. Der Beitrag von Rainer Kirsch wurde bereits zitiert.

40. Czechowski, Heinz: Prometheus. In: Prometheus 1982 (wie Anm. 13), S. 32f. Im Zusammenhang mit der Publikation des Gedichtbandes Kein näheres Zeichen nahm Czechowski einige Veränderungen am Text des Gedichtes vor, deren wichtigste darin besteht, dass er, im Sinne der Goetheschen Ode, die Menschen nicht nach dem Bild der Götter, sondern seinem eigenen schafft: „Also schuf ich dieses Geschlecht/Nach meinem Bilde“; vgl. Czechowski, Heinz: Prometheus. In: Kein näheres Zeichen. Gedichte. Halle/Leipzig 1987, S. 136.

41. Arlt, Peter: Mythos - Phantasie - Wirklichkeit. In: Bildende Kunst. H. 3 (1985), S. 115.

42. Eine Ausnahme machte Rudolf Pakulla, der in einer Diskussionsrunde, veranstaltet von der Zeitschrift Weimarer Beiträge, vom Künstler „das Ende des Schöpferischen“ in Szene gesetzt sehen wollte; vgl. „Prometheus verläßt das Theater“ von Wolfgang Mattheuer. In: Weimarer Beiträge. H. 8 (1983), S. 1381.

\section{RÉSUMÉS}

Dans l'histoire de la culture, la réception du mythe de Prométhée s'est développée principalement selon trois axes, marqués à l'origine par les noms d'Hésiode, d'Eschyle et de Platon. Ceux-ci renvoient essentiellement à des interprétations différentes du mythe. Il s'y ajoute encore une variante de tradition orale, fixée littérairement par Ovide dans les Métamorphoses, où le fils des Titans apparaît comme artisan façonnant des hommes. Dans la phase de constitution de la RDA, c'est d'abord au Prométhée d'Eschyle que l'on fait appel dans l'art et la littérature, avant tout pour figurer le symbole de la libération du prolétariat par lui-même, figure de proue d'une nouvelle société. Avec la désillusion croissante au regard des attentes nourries dans les années 60 , les artistes et les écrivains se tournent vers la tradition transmise dans le dialogue platonicien entre Protagoras et Socrate, où l'accent principal porte sur l'inachèvement de l'œuvre prométhéenne. Les figures de Prométhée des années 80 renvoient pour finir à une situation non seulement de crise de la société, mais aussi de fin du monde. C'est ce que fait apparaître la gravure Prométhée quitte le théâtre, de Wolfgang Mattheuer. Le Prométhée artiste remet ici son propre ouvrage en cause et le livre au feu, ce feu qu'il avait autrefois lui-même apporté.

Among the collection of ancient myths which remain relevant even in the present times, the Prometheus myth occupies an important place. This is based, among other things, on the fact that the figure of Prometheus is characterised by a set of attributes that comprise his ambivalence. The interpretation of this myth in cultural history follows three main lines of tradition which originated with Hesiod, Aeschylus, and Plato. ForHesiod, The theft of the fire by Prometheus, the son of the Titians, plays an essential role, for he is responsible for separating the gods from the humans. In Aeschylus' drama Prometheus Bound, Prometheus is a symbol of the 
human goals of freedom and independence. Plato puts this idea in perspective in his Protagoras dialogue. He claims that Prometheus was only able to endow humans with technical knowledge and skills, but not with statecraft. These last two traditions had a great impact on the interpretation of the Prometheus myth in Eastern Germany including the former GDR. Aeschylus' version was initially dominant in the government of the socialist state, which celebrated itself as the executor of the Promethean will. Following the consolidation of power and especially at the first signs of decay in the state's authority, artists increasingly adopted Plato's view. At this time, the ambivalence of the Promethean influence was emphasised.

Die Rje nezeption des Prometheus-Mythos vollzog sich in der Kulturgeschichte im wesentlichen in drei Traditionslinien, deren Ursprünge die Namen Hesiod, Aischylos und Platon markieren. Sie verweisen signifikant auf unterschiedene Sichtweisen des mythischen Geschehens. Hinzu kommt noch eine mündlich tradierte und schließlich von Ovid in den Metamorphosen schriftlich fixierte Variation des Mythos, in der der Titanensohn als Menschenbildner figuriert. In der Konstitutionsphase der DDR wird in Kunst und Literatur zunächst der aischyleische Prometheus vornehmlich als Symbol der Selbstbefreiung des Proletariats, als Leitbild für eine neue Gesellschaft bemüht. Bedingt durch eine zunehmende Desillusionierung gegenüber den anfänglichen Erwartungshaltungen in den 60ger Jahren des 20. Jahrhunderts wenden sich Künstler und Schriftsteller der von Platon in seinem Protagoras-Sokrates-Dialog vermittelten Traditionslinie $\mathrm{zu}$, in der vor allem die Unfertigkeit prometheischen Tuns hervorgehoben wird. Die Prometheus-Gestaltungen der 80er Jahre verweisen schließlich nicht nur auf eine gesellschaftliche Krisen-, sondern eine Endzeitsituation. Deutlich zeigt dies die Graphik Prometheus verläßt das Theater von Wolfgang Mattheuer. Der Künstler-Prometheus stellt hier sein eigenes Werk in Frage, überantwortet es dem Feuer, welches er zuvor selbst gebracht hatte.

\section{INDEX}

Mots-clés : RDA, mythe de Prométhée

\section{AUTEURS}

\section{HANS JOACHIM KERTSCHER}

Universität Halle/Saale 